\title{
FINITE TOPOLOGICAL SPACES
}

\author{
BY \\ R. E. STONG
}

1. Introduction. The object of this paper is to consider finite topological spaces; i.e. spaces having only a finite number of points. Most of the results obtained are clearly valid for spaces having only a finite number of open sets.

In $\$ 2$, an analysis of the homeomorphism classification of finite spaces is made and a representation of these spaces as certain classes of matrices is obtained. In $\S 3$, the point-set topological properties of finite spaces are considered. The topics covered are separation axioms, connectivity, mappings, and function spaces. In $\$ 4$, the classification of finite spaces by homotopy type is made, reducing homotopy problems back to related homeomorphism problems. In $\S 5$, a classification of finite $H$-spaces is made. It is shown that all such are of the obvious types. In $\$ \sigma$, the homotopy classes of mappings of a finite simplicial complex into a finite space are examined.

General references and definitions may be found in [2], [4], and [5].

The author is greatly indebted to Professor I. M. James for suggesting these questions and for discussions on these topics. The author is also indebted to the National Science Foundation for financial support during this work.

\section{Homeomorphism classification.}

Proposition 1. Let $F$ be a finite topological space with topology $\mathscr{T}$. There exists a unique minimal base $\mathscr{U}$ for the topology.

Proof. For each $x \in F$, let $U_{x}$ be the intersection of all open sets of $F$ which contain $x$. Since $F$ is finite, this is a finite intersection and so $U_{x}$ is open. Let $\mathscr{U}$ be the collection of all $U_{x}$. It is immediate that $\mathscr{U}$ is a base for $\mathscr{T}$ and that any base for $\mathscr{T}$ contains $\mathscr{U}$.

NoTE. Spaces having this property, i.e. that any intersection of open sets is open, were considered by Alexandroff [1].

Proposition 2. Let $F$ be a finite set, $\mathfrak{A}$ a collection of nonempty subsets of $F$. Then $\mathfrak{A}$ is the minimal base for a topology of $F$ if and only if:

(1) $\bigcup\{A \mid . t \in \mathfrak{A}\}=F$,

(2) if $A, B \in \mathfrak{A}$, there exist sets $C_{\alpha} \in \mathfrak{A}$ such that $A \cap B=\bigcup C_{\alpha}$, and

(3) if $A_{\alpha} \in \mathfrak{X}$ and $\bigcup_{\alpha} A_{\alpha} \in \mathfrak{A}$, there exists a $\beta$ such that $A_{\beta}=\bigcup_{\alpha} A_{\alpha}$.

Received by the editors July 16, 1965. 
Proof. Conditions 1 and 2 are the usual conditions that $\mathfrak{U}$ is a base for a topology. If $\mathscr{U}$ is the minimal base, then $\mathscr{U} \subset \mathfrak{A}$. Then if $A \in \mathfrak{A}, A$ is a union of elements of $\mathscr{U}$ since $\mathscr{U}$ is a base, but by condition 3 and $\mathscr{U} \subset \mathfrak{A}, A$ is one of the sets of $\mathscr{U}$ of which it was the union.

If $\mathscr{U}$ is a minimal base, (1) and (2) are trivial and if $U_{x}=\bigcup U_{i}$, then there is a $j$ such that $x \in U_{j}$, so $U_{x} \subset U_{j}$ by definition of $U_{x}$, so that (3) is satisfied.

Proposition 3. Let $F$ and $G$ be finite spaces with minimal bases $\mathscr{U}$ and $\mathscr{V}$. Then:

(a) If $G$ is a subspace of $F, \mathscr{V}=\{U \cap G \mid U \in \mathscr{U}\}$.

(b) If $F \times G$ is the product space, then the minimal base is

$$
\mathscr{U} \times \mathscr{V}=\{U \times V \mid U \in \mathscr{U}, V \in \mathscr{V}\} .
$$

Definition. Let $\mathscr{M}^{\prime}$ be the set of all square matrices $\left(\alpha_{i j}\right)$ with integral entries such that:

(1) $\alpha_{i i} \geqq 1$,

(2) $i \neq j \Rightarrow \alpha_{i j}=-1,0$, or 1 ,

(3) $i \neq j \Rightarrow \alpha_{i j}=-\alpha_{j i}$, and

(4) for any sequence $\left(i_{1}, i_{2}, \cdots, i_{s}\right), s>2, i_{1} \neq i_{2} \neq \cdots \neq i_{s}$ such that

$$
\alpha_{i_{1} i_{2}}=\cdots=\alpha_{i_{s-1} i_{s}}=1, \alpha_{i_{1} i_{s}}=0 .
$$

If $A, B \in \mathscr{M}^{\prime}$ and there is a permutation matrix $T$ with $T A T^{-1}=B$, then say $A$ is equivalent to $B$. Let $\mathscr{M}$ be the set of equivalence classes of elements of $\mathscr{M}^{\prime}$.

THEOREM 1. The homeomorphism classes of finite topological spaces are in one-to-one correspondence with $\mathscr{M}$.

Proof. Let $F$ be a finite topological space with minimal base $\mathscr{U}$. Choose an ordering of $\mathscr{U}$ so $\mathscr{U}=\left\{U_{1}, U_{2}, \cdots, U_{r}\right\}$. Define a matrix $\left(\alpha_{i j}\right) i, j=1, \cdots, r$, by:

$$
\alpha_{i j}=\left\{\begin{array}{l}
\text { Number of } x \in F \ni U_{x}=U_{i} \text { if } j=i, \\
\left\{\begin{array}{c}
1 \text { if } U_{i} \supset U_{j} \text { and } \exists \text { no } k \ni U_{i} \supset U_{k} \supset U_{j} \\
-1 \text { if } U_{j} \supset U_{i} \text { and } \exists \text { no } k \ni U_{j} \supset U_{k} \supset U_{i} \\
0 \text { otherwise }
\end{array}\right\} \text { if } j \neq i .
\end{array}\right.
$$

Trivial verification shows that $\left(\alpha_{i j}\right) \in \mathscr{M}^{\prime}$. Choosing a different order on $\mathscr{U}$ gives a permutation of $\{1, \cdots, r\}$ and hence $F$ determines an element of $\mathscr{M}$.

If $f: F \rightarrow G$ is a homeomorphism, then $f$ induces a map of minimal bases, which preserves inclusions and numbers of elements in each base set, so $F$ and $G$ determine the same element of $\mathscr{M}$.

If $F$ and $G$ give the same element of $\mathscr{M}$ then by proper ordering of bases, they give the same matrix. A homeomorphism is easily constructed, thus showing that the function to $\mathscr{M}$ is monic. 
To see that the function to $\mathscr{M}$ is onto, let $A=\left(\alpha_{i j}\right) \in \mathscr{M}^{\prime}$, with $A$ an $r \times r$ matrix. Let $S=\left\{(a, b) \in Z \times Z \mid 1 \leqq a \leqq r, 1 \leqq b \leqq \alpha_{a a}\right\}$ and for $i=1, \cdots, r$, let

$$
U_{i}=\left\{\begin{array}{ll}
(a, b) \in S \mid \begin{array}{l}
a=i \text { or } a \neq i \text { and } \exists \text { chain }\left(i_{1}, \cdots, i_{s}\right), \\
i=i_{1} \neq i_{2} \neq \cdots \neq i_{s}=a \text { with } \alpha_{i_{1} i_{2}}=\cdots=\alpha_{i_{s}-1 i_{s}}=1
\end{array}
\end{array}\right\} .
$$

It is easy to verify that the collection of $U_{i}$ is a minimal base for a topology on $S$, and that with this topology $S$ gives the matrix $A$.

It should be noted that the size $(r \times r)$ of the matrix is the number of sets in the minimal base and that the trace $\left(\sum \alpha_{i i}\right)$ of the matrix is the number of points in the space.

3. Point-set topology. First, it is clear that if a finite space is $T_{1}$ then it is in fact discrete. However, $T_{0}$-spaces are more useful and one has:

Proposition 4 (AleXandroff [1]). A finite space $F$ is $T_{0}$ if and only if $U_{x}=U_{y}$ implies $x=y$.

Note. Thus $F$ is $T_{0}$ if and only if its matrix has only 1 's on the diagonal.

Proof. If $F$ is $T_{0}$ and $x \neq y$, there is an open set $V$ such that $x \in V, y \notin V$ (or $y \in V, x \notin V$ ). Thus $y \notin U_{x}$ (or $x \notin U_{y}$ ) so $U_{x} \neq U_{y}$. Conversely, if $U_{x}=U_{y}$ implies $x=y$ and $u, v \in F$ such that every open set containing one contains the other, then $v \in U_{u}, u \in U_{v}$, so $U_{u}=U_{v}$ and $u=v$.

For $T_{0}$-spaces, the partial order by inclusion on $\mathscr{U}$ gives a partial order on the space, and thus $x \leqq y$ is equivalent to $U_{x} \subset U_{y}$ or $x \in U_{y}$. For non- $T_{0}$-spaces, one may also write $x \leqq y$ for $x \in U_{y}$, and the proposition then becomes: $F$ is $T_{0}$ if and only if $x \leqq y$ and $y \leqq x$ implies $y=x$.

Now, consider connectivity. First, each set $U_{x}$ is connected, for if $A, B$ are open, $U_{x} \subset A \cup B$, then $x$ is in one set, say $x \in A$ and so $U_{x} \subset A$. Thus a finite space is locally connected. The usual open-closed argument shows:

Proposition 5. Let $F$ be a connected finite space, and $x$ and $y$ in $F$. Then there exists a sequence $z_{0}, z_{1}, \cdots, z_{s}$ of elements of $F$ such that $z_{0}=x, z_{s}=y$ and for each $i(0 \leqq i \leqq s-1)$, either $z_{i} \leqq z_{i+1}$ or $z_{i} \geqq z_{i+1}$.

Proposition 6. Let $F$ be a finite space, $x, y \in F$ and $x \leqq y$. Then there is $a$ path in $F$ whose ends are $x$ and $y$.

Proof. Let

$$
\phi: I=[0,1] \rightarrow F \text { by } \phi(t)=\left\{\begin{array}{l}
y t=0 \\
x t>0
\end{array} .\right.
$$

If $V$ is any open set in $F$ and

(1) $y \in V$, then $x \leqq y$ so $x \in U_{y} \subset V$ and $\phi^{-1}(V)=I$.

(2) $x \in V, y \notin V$, then $\phi^{-1}(V)=(0,1]$.

(3) $x \notin V$, then $\phi^{-1}(V)=\varnothing$. 
Thus $\phi$ is continuous.

Combining Propositions 5 and 6, one has immediately:

COROLlary 1. Connectedness and path-connectedness are equivalent for finite spaces.

Next, consider functions.

Proposition 7. Let $F$ and $G$ be finite spaces. $f: F \rightarrow G$ a function. $f$ is continuous if and only if $x \leqq y$ implies $f(x) \leqq f(y)$.

Proof If $f$ is continuous, $x \leqq y$, then $U_{f(y)}$ is open so $f^{-1}\left(U_{f(y)}\right)$ is open and contains $y$. Thus it contains $U_{y}$ and hence $x\left(x \in U_{y}\right)$. Thus $f(x) \in U_{f(y)}$ or $f(x) \leqq f(y)$. Conversely, suppose $x \leqq y$ implies $f(x) \leqq f(y)$, and let $V$ be open in $G$. If $y \in f^{-1}(V), f(y) \in V$ so $U_{f(y)} \subset V$. Then if $x \in U_{y}, x \leqq y$ and so $f(x) \leqq f(y)$ or $f(x) \in U_{f(y)}$. Thus $f(x) \in V$ or $x \in f^{-1}(V)$. Since $f^{-1}(V)=\bigcup_{y \in f_{-1}(V)} U_{y}$, it is open and so $f$ is continuous.

Proposition 8. Let $F$ be a finite space, $f$ a continuous map of $F$ into itself. If $f$ is either one-to-one or onto, then it is a homeomorphism.

Proof. Since $F$ is finite, one-to-one and onto are equivalent. Since $f$ is oneto-one, $A \rightarrow f(A)$ defines a 1-1 correspondence $f^{\prime}: 2^{F} \rightarrow 2^{F}$. If $f^{\prime}(A)$ belongs to the topology $\mathscr{T} \subset 2^{F}, f(A)$ is open and by continuity and the 1-1 nature of $f, A \in \mathscr{T}$. Since $\mathscr{T}$ is finite and $f^{\prime}(\mathscr{T}) \supset \mathscr{T}, f^{\prime}$ gives a $1-1$ correspondence $\mathscr{T} \rightarrow \mathscr{T}$. Thus $A$ open implies $f(A)$ open, and $f^{-1}$ is continuous.

Finally, consider spaces of maps. Let $X$ be any topological space, $F$ a finite space and let $F^{X}$ denote the space of continuous maps of $X$ into $F$, with the compact open topology. If $f, g \in F^{X}$, write $g \leqq f$ if $g(x) \leqq f(x)$ for all $x \in X$.

Proposition 9. The intersection of all open sets in $F^{X}$ containing the map $f$ is $\left\{g \in F^{X} \mid g \leqq f\right\}$.

Proof Let $V_{f}$ be the intersection of all open sets containing $f$ and $W_{f}=\left\{g \in F^{X} \mid g \leqq f\right\}$. For any compact set $K \subset X$ and open set $U \subset F$, let $(K, U)=\left\{g \in F^{X} \mid g(K) \subset U\right\}$. If $g \in V_{f}$ and $x \in X$, then $x$ is compact, $U_{f(x)}$ is open and $f \in\left(\{x\}, U_{f(x)}\right)$. Thus $g \in\left(\{x\}, U_{f(x)}\right)$ or $g(x) \in U_{f(x)}$. Thus $g(x) \leqq f(x)$ for any $x \in X$, or $g \in W_{f}$. If $g \in W_{f}$ and $(K, U)$ is any sub-basic open set of $F^{X}$ containing $f$, then for all $x \in K, f(x) \in U$. Then $g(x) \leqq f(x)$ or $g(x) \in U_{f(x)}$. Since $U$ is open and contains $f(x)$, it contains $U_{f(x)}$. Thus $g(x) \in U$ for all $x \in K$, or $g \in(K, U)$. Thus $g$ belongs to all open sets containing $f$ or $g \in V_{f}$.

Note. If $X$ is also a finite space, $F^{X}$ is finite and the order on functions is the same as the order given by the compact open topology.

One has the standard result (see [3]):

LEMMA 1. Let $X$ and $Y$ be topological spaces. Suppose that for all $p \in X$ the 
compact neighborhoods of p form a base for the neighborhood system of $p$. Then:

(a) If $\phi: X \times I \rightarrow Y$ is continuous, so is $\phi^{\prime}: I \rightarrow Y^{X}: t \rightarrow \phi(, t)$.

(b) If $\psi: I \rightarrow Y^{X}$ is continuous, so is $\bar{\psi}: X \times I \rightarrow Y:(x, t) \rightarrow \psi(t)(x)$.

In particular, these conditions are satisfied if $X$ is a finite space.

Thus one has:

COROLlaRY 2. If $F$ and $G$ are finite spaces, the homotopy classes of maps of $F$ to $G$ are in one-to-one correspondence with the components of $G^{F}$.

Corollary 3. If $f, g: F \rightarrow G$ and $f \leqq g$, then $f$ is homotopic to $g$ by a homotopy which keeps pointwise fixed the set $\{x \in F \mid f(x)=g(x)\}$.

Proof. Since $G^{F}$ is finite, Proposition 6 applies and applying (b) of the lemma to the path given produces the desired homotopy.

\section{Homotopy-type classification.}

Definition. Let $F$ be a finite space

(a) $x \in F$ is linear if $\exists y>x \ni z>x$ implies $z \geqq y$,

(b) $x \in F$ is colinear if $\exists y<x \ni z<x$ implies $z \leqq y$.

Definition. A finite space $F$ (with base point $p$ ) will be called a core if $F$ is $T_{0}$ and has no linear or colinear points (except possibly $p$ ).

Definition. A core of the finite space $F$ (with base point $p$ ) is a subspace $F_{1}$ of $F$ (with the same base point) such that $F_{1}\left(\left(F_{1}, p\right)\right)$ is a core and such that $F_{1}$ is a strong deformation retract of $F$.

Theorem 2. Let $F$ be a finite space (with base point $p$ ). Then $F$ has a core.

Proof. (a) Let $\mathscr{U}$ be the minimal base for the topology of $F$, and for each $U \in \mathscr{U}$, let $x_{U} \in U$ such that $U_{x_{U}}=U$ (choosing $p$ for $U_{p}$ ). Let $F^{\prime}$ be the subspace of all $x_{U}$. For each $x \in F$, let $f(x)=x_{U}$ where $U=U_{x}$. Then $x_{U} \in U_{x}$ or $x_{U}=f(x) \leqq x$. If $x \leqq y, x \in U_{y}$ so $f(x) \in U_{x} \subset U_{y}=U_{f(y)}$ or $f(x) \leqq f(y)$. Thus $f$ is continuous and $f \leqq$ identity.

By Corollary $3, f$ is homotopic to the identity by a homotopy fixing $F^{\prime}$, so $F^{\prime}$ is a strong deformation retract of $F$. Further $F^{\prime}$ is $T_{0}$.

(b) Let $F$ be a finite space, $x \in F$ a linear point. Then $F-x$ is a strong deformation retract of $F$. To see this, let $f: F \rightarrow F$ by $f(z)=z$ if $z \neq x, f(x)=y$, where $y>x$ such that $z>x$ implies $z \geqq y$. If $u \leqq v$ :

(1) $u=x, v=x$, then $f(u)=f(v)$,

(2) $u=x, v \neq x$, then $f(u)=y, f(v)=v$ and $v>x$ so $v \geqq y$ giving $f(u) \leqq f(v)$,

(3) $u \neq x, v=x$, then $f(u)=u \leqq x<y=f(v)$, and

(4) $u \neq x, v \neq x$, then $f(u)=u \leqq v=f(v)$.

Thus $f$ is continuous and clearly $f \geqq$ identity. By Corollary $3, f$ is homotopic to the identity by a homotopy fixing $F-x$.

By reversing the inequalities, one has 
(c) Let $F$ be a finite space, $x \in F$ a colinear point, then $F-x$ is a strong dcformation retract of $F$.

(d) Now let $F$ be any space and form subspaces $G_{i}$ of $F$ by:

(1) $G_{1}=F^{\prime}$ as in (a),

(2) if $G_{i}$ is defined and has a point $x(\neq p)$ which is linear or colinear, let $G_{i+1}=G_{i}-x$.

Since $F$ is finite, this process must terminate. Let $F_{1}$ be the terminal subspace. $F_{1}$ is $T_{0}$, being a subspace of $F^{\prime}$ and has no linear or colinear points (other than perhaps $p$ ). Thus $F_{1}$ (or $\left(F_{1}, p\right)$ ) is a core. Since each $G_{i}$ is a strong deformation retract of $G_{i-1}$, and $G_{1}$ is a strong deformation retract of $F, F_{1}$ is a strong deformation retract of $F$.

Thus $F_{1}$ is a core of $F$.

THEOREM 3. Let $F($ or $(F, p)$ ) be a core. Then any map $f: F \rightarrow F$ (preserving base point) which is homotopic to the identity (relative to base points) is the identity.

Proof Let $f: F \rightarrow F(f(p)=p)$ and suppose $f \geqq$ identity. Then for all $x \in F$, $f(x) \geqq x$. If $x$ is a maximal point of $F$, then $f(x) \geqq x$ implies $f(x)=x$. Then suppose $x \in F$ and for all $z>x, f(z)=z$. Then for all $z>x, z=f(z) \geqq f(x)$ by continuity of $f$. Since $f(x) \geqq x$, either $x$ is linear or $f(x)=x$. Since the only possible linear point is $p$, and $f(p)=p$, this gives $f(x)=x$. By induction, $f$ is the identity.

Similarly, $f \leqq$ identity implies $f=$ identity.

By Proposition 5, the component of the identity in $F^{F}$ (or the subspace of base point preserving maps) is a single map, the identity. By the relation between homotopy and components, this gives that any $f$ homotopic to the identity must be the identity.

THEOREM 4. Let $F, G$ be finite spaces (with base points $p$ and $q$ ) and with cores $F_{1}, G_{1}$. Then $F$ is homotopy equivalent to $G$ if and only if $F_{1}$ is homeomorphic to $G_{1}$ (relative to base points).

Proof. Since a core of a space is a strong deformation retract, it has the same homotopy type as the space. Thus if $F_{1}$ and $G_{1}$ are homeomorphic, both $F$ and $G$ have the same homotopy type as $F_{1}$. Also, if $F$ and $G$ have the same homotopy type, $F_{1}$ is homotopy equivalent to $G_{1}$. Let $f: F_{1} \rightarrow G_{1}, g: G_{1} \rightarrow F_{1}$ be a homotopy equivalence. Then $g f$ and $f g$ are homotopic to the identities. By Theorem 3, they are equal to the identities, and so $g=f^{-1}$ and $f, g$ are homeomorphisms.

REMARKS. (1) By Theorem 4, it is meaningful to talk of "the core" of a finite space, as the homeomorphism class of the cores of the space.

(2) In any homotopy equivalence class of finite spaces there is a representative with a minimal number of points. This minimal space is a core and its homeomorphism class is the core of any space in the homotopy class. 
(3) If $F$ is a finite space, the cores of $F$ are the minimal strong deformation retracts of $F$ in the partial order by inclusion.

CoROllary 4. Let $F$ be a finite space. Then $F$ is contractible if and only if some point of $F$ is a strong deformation retract of $F$.

Proof. $F$ contractible is equivalent to $F$ has the homotopy type of a point, which is equivalent to the core of $F$ being a point.

5. Finite $H$-spaces. There are two definitions of $H$-space which will be considered here.

Definition. An $H$-space (of type $\mathrm{I}$ ) is a triple $(F, p, \mu)$ where $F$ is a space, $p \in F$ is a base point and $\mu: F \times F \rightarrow F$ is a map such that

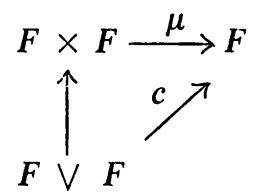

commutes up to homotopy, where $F \vee F=\{(x, y) \in F \times F \mid x=p$ or $y=p\}$ and $c$ is the collapse, $c(x, p)=x, c(p, x)=x$.

As is obvious, if $G$ is any space, $G \cup p=F$ (disjoint union) admits an $H$-space structure. It will be shown that all finite $H$-spaces of type I are equivalent to one having this form: i.e. the component of the base point is contractible.

Definition. An $H$-space (of type II) is a space $F$ (with base point $p$ ) and a map $\mu: F \times F \rightarrow F$ such that

and

$$
\mu_{1}: F \times F \rightarrow F \times F:(x, y) \rightarrow(x, \mu(x, y)),
$$

$$
\mu_{2}: F \times F \rightarrow F \times F:(x, y) \rightarrow(y, \mu(x, y))
$$

are homotopy equivalences.

Definition. Two $H$-spaces $(F, \mu),(G, v)$ (with base points) are said to be equivalent if there exists a homotopy equivalence $f: F \rightarrow G, g: G \rightarrow F$ such that the diagrams

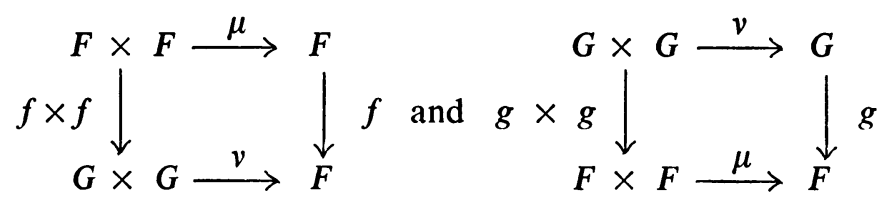

commute up to homotopy.

Proposition 10. If $F$ and $G((F, p)$ and $(G, q))$ are homotopy equivalent, there is a one-to-one correspondence between the $H$-space structures on $F$ and those on $G$. 
Since any finite space is homotopy equivalent to its core, one may consider first the situation for cores. Temporarily, all spaces considered will be with base point.

Proposition 11. Let $(F, p, \mu)$ be a finite $H$-space (either type) and let $(F, p)$ be a core. Then the maps

$$
\theta_{1}: F \rightarrow F: x \rightarrow \mu(x, p)
$$

and

$$
0_{2}: F \rightarrow F: x \rightarrow \mu(p, x)
$$

are homeomorphisms.

Proof. If the $H$-space is of type I, one has

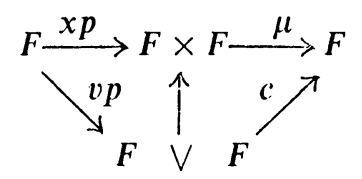

commutative up to homotopy, or $\theta_{1}$ is homotopic to the identity, hence is the identity by Theorem 3 .

If the $H$-space is of type II, let $\phi_{2}$ be the homotopy inverse of $\mu_{2}$. One has

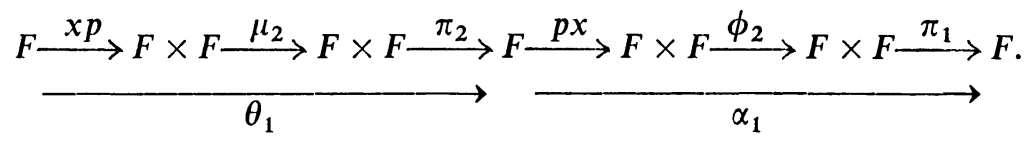

Now, on the image of $F,(p x) \circ \pi_{2}$ is the identity, so

$$
\alpha_{1} \theta_{1}=\pi_{1} \circ \phi_{2} \circ \mu_{2} \circ(x p) \sim \pi_{1} \circ(x p)=\text { identity. }
$$

Since $(F, p)$ is a core, $\alpha_{1} \theta_{1}$ is the identity. Since $\theta_{1}$ is then one-to-onc, it is a homeomorphism by Proposition 8 .

Proposition 12. Let $(F, p)$ be a core, $x \in F$. Then:

(1) $x$ is less than two distinct maximal points, or

(2) $x$ is maximal, or

(3) $x$ is linear under a maximal point; hence $x=p$; and

(1') $x$ is greater than two distinct minimal points, or

(2') $x$ is minimal, or

(3') $x$ is colinear over a minimal point; hence $x=p$.

Proof. Let $A$ be the set of points not satisfying (1), (2) or (3), and let $x$ be a maximal element of $A$. Since $x \in A, x$ is not maximal in $F$ and there is a $z>x$. Let $B=\{z \mid z>x\} \neq \varnothing$. If any element of $B$ satisfies (1), then $x$ satisfies (1). If $p \notin B$, then every element of $B$ is maximal, and since $B$ doesn't contain two 
maximals, $B$ must consist of one point, $z$. Then $w>x \Rightarrow w=z$, so $x$ is linear under a maximal and satisfies (3). Thus $p \in B$. Since $p>x, x$ is not linear, and there is a $y>x$ such that $y$. Since $y$ is not type (1) or (3), $y$ is maximal. Let $w$ be maximal with $w \geqq p$. Then $y, w$ are maximal, $y, w>x$ and since $w \geqq p, y \geqq p$, $w \neq y$.

Hence $x$ satisfies (1), but $x \in A$. Thus $A$ is empty.

Proposition 13. Let $(F, p)$ be a core, $(F, p, \mu)$ an $H$-space (either type). Then $p$ is both maximal and minimal.

Proof. (a) $p$ doesn't satisfy (1) or $\left(1^{\prime}\right)$.

If $m, m^{\prime}$ are maximals, $m, m^{\prime}>p$, then since $\mu(, p)$ is a homeomorphism, $\mu(m, p)$ is maximal, and since $\mu(p$,$) is a homeomorphism, \mu\left(p, m^{\prime}\right)$ is maximal. Since $m^{\prime}>p,\left(m, m^{\prime}\right)>(m, p)$ and so by continuity of $\mu, \mu\left(m, m^{\prime}\right) \geqq \mu(m, p)$. Since $m>p,\left(m, m^{\prime}\right)>\left(p, m^{\prime}\right)$ and $\mu\left(m, m^{\prime}\right) \geqq \mu\left(p, w^{\prime \prime}\right)$. By maximality, $\mu\left(p, m^{\prime}\right)$ $=\mu\left(m, m^{\prime}\right)=\mu(m, p)$. Taking $m^{\prime}=m, \mu(p, m)=\mu(m, n)=\mu(m, p)$, and so for any $m, m^{\prime}, \mu\left(p, m^{\prime}\right)=\mu(m, p)=\mu(p, m)$. Since $\mu(p$,$) is 3$ homeomorphism, this gives $m=m^{\prime}$.

Similarly, $p$ cannot satisfy $\left(1^{\prime}\right)$.

(b) $p$ doesn't satisfy $\left(2^{\prime}\right)$ and (3).

Suppose $p$ satisfies $\left(2^{\prime}\right)$ and (3).

For $r \geqq 0, F$ contains a subset $D_{r}=\left\{p=u_{0}, u_{1}, \cdots, u_{r} ; m_{0}, \cdots, m_{r-1}\right\}$ with $u_{i}$ minimal, $m_{i}$ maximal in $F$ and such that

(1) $m_{i}>x$ if and only if $x=u_{i}$ or $u_{i+1}(i=0, \cdots, r-1)$,

(2) $y>u_{0}$ if and only if $y=m_{0}$,

(3) $y>u_{i}(i=1, \cdots, r-1)$ if and only if $y=m_{i-1}$ or $m_{i}$,

(4) $\mu\left(x, m_{i}\right)=\mu\left(m_{i}, x\right)=m_{i}$ if $x=m_{k}$ or $u_{k}, k \leqq i$ and $1 \leqq i \leqq r-1$.

(5) $\mu\left(x, u_{i}\right)=\mu\left(u_{i}, x\right)=u_{i}$ if $x=m_{k}, k<i$ or $x=u_{k}, k \leqq i$ and $0 \leqq i \leqq r$.

(6) $\mu\left(x, m_{i}\right)=\mu(x, p)=\mu\left(x, u_{i}\right) ; \mu\left(m_{i}, x\right)=\mu(p, x)=\mu\left(u_{i}, x\right)$ for all $x \notin D_{r}$.

For $r=0$, this is true; since then $D_{0}=\left\{p=u_{0}\right\}$ and one has only to verify that $p$ is minimal and that $\mu(p, p)=p$.

Assuming $F$ contains $D_{r}$, one can show it must contain $D_{r+1}$.

First, $m_{r}$ exists. If $r=0$, by assumption, there is a unique $x>p$, which is a maximal, $m_{0}$. If $r>0$ there is a maximal $m_{r}>u_{r}, m_{r} \notin D_{r}$, for $u_{r} \neq p$, and $u_{r}$ is not maximal $\left(u_{r}<m_{r-1}\right)$ so $u_{r}$ is less than two maximals, only one being in $D_{r}$. If there are two maximals, $m, m^{\prime} \notin D_{r}$ with $m, m^{\prime}>u_{r}$, then $\mu\left(m, u_{r}\right)=\mu(m, p)$ is maximal $\left(\mu(, p)\right.$ a homeomorphism) $\mu\left(m, u_{r}\right) \geqq \mu\left(u_{r}, u_{r}\right)=u_{r}$ (continuity of $\mu$ ) $\mu\left(m, u_{r}\right) \notin D_{r}$ for $\mu(, p)$ is a homeomorphism. As in (a), $\mu\left(m, u_{r}\right)=\mu\left(u_{r}, m\right)$ $=\mu(m, m)=\mu\left(m, m^{\prime}\right)=\mu\left(m^{\prime}, u_{r}\right)=\mu\left(u_{r}, m^{\prime}\right)=\mu(m, p)=\mu\left(m^{\prime}, p\right)$ and being maximals over $u_{r}$, all are equal to $m$, or $m^{\prime}$.

There is no $z$ with $u_{r}<z<m_{r}$, else $z$ is not maximal and $z \neq p$, so $z$ would lie under two maximals. Since $u_{r}$ can lie under only $m_{r-1}$ and $m_{r}, z<m_{r-1}$, contradicting assumption (1) for $D_{r}$. 
Assumption (6) verifies the multiplication rule (4) for $m_{r}$, and one need only verify (6) for $m_{r}$. For this, let $\theta: F \rightarrow F: x \rightarrow \mu\left(x, m_{r}\right)$ (or $\mu\left(m_{r}, x\right)$ ). If $x \notin D_{r}$, $\theta(x)=\mu\left(x, m_{r}\right) \geqq \mu\left(x, u_{r}\right)=\mu(x, p)$ (or $\theta(x) \geqq \mu(p, x)$ ). If $x$ is maximal, so is $\mu(x, p)$ (or $\mu(p, x)$ ) and $\theta(x)=\mu(x, p)$ (or $\mu(p, x))$. Then if for all $y>x, \theta(y)$ $=\mu(y, p)$, and if $z>\mu(x, p)$; since $\mu(, p)$ is a homeomorphism, there is a $z^{\prime}>x$, with $\mu\left(z^{\prime}, p\right)=z$. Then $z^{\prime}>x \Rightarrow z^{\prime} \notin D_{r}$ and so $z=\theta\left(z^{\prime}\right) \geqq \theta(x)$. Since $\mu(x, p) \neq p$, $\mu(x, p)$ is not linear and so $\theta(x)=\mu(x, p)$.

Since $m_{r} \neq p, m_{r}$ not minimal, there is a $u_{r+1}<m_{r}, u_{r+1}$ minimal, $u_{r+1} \notin D_{r}$. Applying the same arguments as with $m_{r}$, except for reversed inequalities, verifies all hypotheses (1)-(6).

By induction $F$ contains $D_{r}$ for all $r \geqq 0$. Since $F$ is finite, this is a contradiction.

(c) $p$ doesn't satisfy (2) and ( $\left.3^{\prime}\right)$.

By reversal of inequalities in arguments for (b), one can construct a countable set in $F$.

(d) $p$ doesn't satisfy (3) and (3').

For if it did, one can find a set $D_{r}^{\prime} \subset F$,

$$
D_{r}^{\prime}=\left\{z_{0}=p, z_{1}, \cdots, z_{r} ; u_{0}, \cdots, u_{r-1} ; m_{0}, \cdots, m_{r-1} x_{(r-1)}\right\}
$$

with $u_{i}$ minimal, $m_{i}$ maximal and such that

(1) $m_{i}>x$ if and only if $x \leqq z_{i}$ or $z_{i+1}, 0 \leqq i \leqq r-1$.

(2) $u_{i}<x$ if and only if $x \geqq z_{i}$ or $z_{i+1}, 0 \leqq i \leqq r-1$.

(3) $x>z_{0}$ if and only if $x=m_{0} ; x<z_{0}$ if and only if $x=u_{0}$.

(4) $x>z_{i}$ if and only if $x=m_{i}$ or $m_{i-1}, 1 \leqq i \leqq r-1$,

$x<z_{i}$ if and only if $x=u_{i}$ or $u_{i-1}, 1 \leqq i \leqq r-1$.

(5) $m_{r-1}>z_{r}>u_{r-1}$.

(6) $\mu\left(x, z_{i}\right)=\mu\left(z_{i}, x\right)=z_{i}$ if $x=m_{k}, u_{k} ; k<i$ or $x=z_{k}, k \leqq i ; 0 \leqq i \leqq r$.

$$
\mu\left(x, m_{i}\right)=\mu\left(m_{i}, x\right)=\left\{m_{i} \text { if } x=m_{k}, z_{k}, k \leqq i ; x=u_{k}, k<i ; z_{i} \text { if } x=u_{i}\right\}
$$

and

$$
\mu\left(x, u_{i}\right)=\mu\left(u_{i}, x\right)=\left\{u_{i} \text { if } x=u_{k}, z_{k}, k \leqq i ; x=m_{k}, k<i ; z_{i} \text { if } x=m_{i}\right\}
$$

for $0 \leqq i \leqq r-1$.

(7) For $x \notin D_{r}^{\prime}, \mu\left(x, m_{i}\right)=\mu\left(x, z_{i}\right)=\mu\left(x, u_{i}\right)=\mu(x, p)$ and $\mu\left(m_{i}, x\right)=\mu\left(z_{i}, x\right)$ $=\mu\left(u_{i}, x\right)=\mu(p, x)$.

Clearly $D_{0}^{\prime}=\left\{p=z_{0}\right\} \subset F$ and the argument is by induction on $r$, similar to (b). First $m_{r}$ and $u_{r}$ exist as a maximal over $z_{r}$ and a minimal under it. The standard argument gives uniqueness. The relations $\mu(x, m) \geqq \mu\left(x, z_{r}\right) \geqq \mu\left(x, u_{r}\right)$ give all of the multiplication table except $\mu\left(u_{r}, m_{r}\right)=\mu\left(m_{r}, u_{r}\right)=z_{r+1}$. This will in fact define $z_{r+1}$, once equality is known.

Since $u_{r} \neq p, u_{r}$ is not linear and there is a $z>u_{r} \ni z \geq z_{r}$. Choose a minimal such $z$ and call it $z_{r+1}$. Since $m_{r} \neq p, m_{r}$ is not colinear and there is a $w<m_{r} \ni w$ $\$ z_{r}$. Choose a maximal such $w$ and call it $w_{r+1}$. By choice of $z_{r+1}, w_{r+1}, z_{r+1} \notin D_{r}^{\prime}$, 
$w_{r+1} \notin D_{r}^{\prime}$ and there can be no $h$ or $k$ such that $u_{r}<h<z_{r+1}$ or $m_{r}>k>w_{r+1}$.

One then has the inequalities

and

$$
\mu\left(w_{r+1}, p\right) \leqq \mu\left(w_{r+1}, u_{r}\right) \leqq \mu\left(m_{r}, u_{r}\right) \leqq \mu\left(m_{r}, z_{r+1}\right)=\mu\left(p, z_{r+1}\right)
$$

$$
u_{r}=\mu\left(z_{r}, u_{r}\right) \leqq \mu\left(m_{r}, u_{r}\right) \leqq \mu\left(m_{r}, z_{r}\right)=m_{r}
$$

so $\mu\left(m_{r}, u_{r}\right)$ lies between $\mu\left(w_{r+1}, p\right)$ and $\mu\left(m_{r}, p\right)=m_{r}$ and between $u_{r}=\mu\left(p, u_{r}\right)$ and $\mu\left(p, z_{r+1}\right)$. Since nothing lies properly between $u_{r}$ and $z_{r+1}$ or $m_{r}$ and $w_{r+1}$, this is only possible if $\mu\left(m_{r}, u_{r}\right)=\mu\left(p, z_{r+1}\right)=\mu\left(w_{r+1}, p\right)$. Uniqueness of $z_{r+1}$ and $w_{r+1}$ follow and then clearly $z_{r+1}=w_{r+1}=\mu\left(m_{r}, u_{r}\right) . z_{r+1}=\mu\left(u_{r}, m_{r}\right)$ is obtained by the same reasoning.

(e) Combining steps (a)-(d) with Proposition 12, it follows that $p$ is both maximal and minimal.

THEOREM 5. Let $F$ be a finite space. A necessary and sufficient condition that there exists an $H$-space of type $\mathrm{I}(F, p, \mu)$ is that $p$ be a strong deformation retract of its component in $F$. A necessary and sufficient condition that there is an $H$-structure of type I on $F$ for some base point is that a component of $F$ is contractible.

Proof. By Proposition 13, $p$ is a component of the core of $F$ and hence $p$ is the core of its component.

THEOREM 6. Let $F$ be a finite space. A necessary and sufficient condition that there exists an $H$-space of type II $(F, \mu)$ is that every component of $F$ be contractible. A necessary and sufficient condition that there exists an $\mathrm{H}$-space of type II $(F, \mu)$ with base point $p$ is that every component of $F$ is contractible and that $p$ is a strong deformation retract of its component.

Proof. If $p \in F$ is a base point, let $\left(F_{1}, p\right)$ be a core at $p$. By Proposition $13, p$ is a component of $F_{1}$ and hence is a strong deformation retract of its component. Further $p$ is neither linear nor colinear, so $F_{1}$ is a core of $F$. (As well as $\left(F_{1}, p\right)$ being a core of $(F, p)$.)

Then by Proposition 10, with or without base point, there is an $H$-space $\left(F^{\prime}, \mu^{\prime}\right)$ where $F^{\prime}$ is a core of $F$. Analogous to Proposition 10, for any $z \in F^{\prime}$, the maps

$$
\begin{aligned}
& \theta_{1}: F^{\prime} \rightarrow F^{\prime}: x \rightarrow \mu^{\prime}(x, z), \\
& \theta_{2}: F^{\prime} \rightarrow F^{\prime}: x \rightarrow \mu^{\prime}(z, x),
\end{aligned}
$$

are homeomorphisms. Let $m$ be any maximal point of $F^{\prime}, u$ any minimal point. Then $\mu^{\prime}(m, u)$ is maximal, since $m$ is and $\mu^{\prime}(, u)$ is a homeomorphism, and is minimal since $u$ is and $\mu^{\prime}(m$,$) is a homeomorphism. Thus since \mu^{\prime}(, u)$ is a homeomorphism, $m$ is minimal, and since $\mu^{\prime}(m$,$) is a homeomorphism, u$ is maximal. Thus $F^{\prime}$ is discrete, or every component of $F$ is contractible. 


\section{Mappings of a finite complex.}

Proposition 14. Let $X$ be any topological space and $F$ a finite space. If $f, g \in F^{X}$ with $g \geqq f$, then $f$ and $g$ are homotopic.

Proof. Let $H: X \times I \rightarrow F$ be given by $H(x, t)=f(x)$ if $t>0$, and $H(x, t)=g(x)$ if $t=0$. Let $U$ be any open set in $F$. For any $(x, t) \in H^{-1}(U)$, there is a neighborhood $V$ of $(x, t)$ contained in $H^{-1}(U)$. If $t>0, V=f^{-1}(U) \times(0,1]$ and if $t=0$, $V=g^{-1}(U) \times[0,1]$. Thus $H$ gives the required homotopy.

The remainder of this section will be devoted to the study of mappings of a finite simplicial complex into a finite space, with emphasis on relating homotopic maps by means of inequalities. Another approach to the study of such homotopy classes is contained in the work of McCord [6], by which one has for any complex $K$ an associated finite space $F$ and a map $f: K \rightarrow F$ inducing isomorphisms of homotopy groups.

LEMMA 2. Let $K$ be a finite simplicial complex, L a closed subcomplex, $F$ a finite space, $p \in F$, and $f:(K, L) \rightarrow(F, p)$ a continuous function. Then there exist closed subsets

$$
K^{0}=K \supset K^{1} \supset K^{2} \supset \cdots \supset K^{r} \supset K^{r+1}=\varnothing,
$$

finite collections $\Sigma^{i}, i=0, \cdots, r$, of closed subsets $B$ of $K^{i}$ with $f$ constant valued on $B$, and closed subsets $K_{B}$ of $K^{i}$ for $B \in \Sigma^{i}$, such that:

(1) $L \neq \varnothing$ implies $L \in \Sigma^{0}$;

(2) $K_{B}$ is a neighborhood of $B$ in $K^{i}\left(B \in \Sigma^{i}\right)$ with $K_{B} \subset\{z \in K \mid f(z) \leqq f(B)\}$;

(3) $K^{i}=\cup K_{B}$ for $B \in \Sigma^{i}$;

(4) If $B, B^{\prime}$ are distinct elements of $\Sigma^{i}$ and $y \in K_{B} \cap K_{B^{\prime}}$, then $y$ belongs to the boundary in $K^{i}$ of $K_{B}$.

(5) $K^{i+1}=\left\{y \in K^{i} \mid \exists B \neq B^{\prime}\right.$ in $\Sigma^{i}$ with $\left.y \in K_{B} \cap K_{B^{\prime}}\right\}$; and

(6) If $B \in \Sigma, C \in \Sigma^{i}, j>i$, and $K_{B} \cap K_{C} \neq \varnothing$, then $f(C) \leqq f(B)$.

Proof. In order to show this, a construction will be given to produce $\Sigma^{0}$, the sets $K_{B}$ for $B \in \Sigma^{0}$, and the subset $K^{1}$, together with a specific structure of $K^{1}$ as a finite simplicial complex of dimension less than that of $K$. Taking $L^{1}$ to be empty, one then performs the same construction on $K^{1}$. Iteration then gives the sets $K^{i}$, collections $\Sigma^{i}$, and sets $K_{B}$. Since $\operatorname{dim} K^{i+1}<\operatorname{dim} K^{i}$, this process must terminate, giving all objects of the lemma. It will be clear from the construction that all parts of the lemma are valid, except for part (6), which will be proved last.

To begin the construction, let $V$ be an open set containing $L$ and having closure contained in the set $U=\{z \in K \mid f(z) \leqq p\}$ (if $L=\varnothing$, take $V=\varnothing$ ). For each point $x \in K$, let $V_{x}$ be an open set containing $x$ and having closure contained in both $U_{x}=\{z \in K \mid f(z) \leqq f(x)\}$ and the open star $S_{x}$ of $x$ in $K$ (i.e. $S_{x}$ is the interior of the set $\cup \bar{\sigma}$ for all $x \in \bar{\sigma}, \sigma$ a simplex of $K$ ). 
Let $A$ be a finite set of points of $K-L$ for which the sets $V_{x}, x \in A$, cover $K-V$. Let $\Sigma^{0} \subset 2^{K}$ be the finite collection consisting of the sets $\{x\}$ for $x \in A$ and the set $L$ if $L \neq \varnothing$. Clearly $f$ is constant valued on each set $B \in \Sigma^{0}$.

Then $\Sigma^{0}$ is a finite collection of disjoint closed subsets of $K$, so there exist disjoint open sets $W_{B}, B \in \Sigma^{0}$, with $B \subset W_{B}$.

There is then an integer $n$ such that for any closed simplex $\bar{\sigma}$ of the $n$th barycentric subdivision $K(n)$ of $K$ :

(a) $\bar{\sigma} \cap \bar{V} \neq \varnothing$ implies $\bar{\sigma} \subset U$,

(b) $\bar{\sigma} \cap \bar{V}_{x} \neq \varnothing, x \in A$, imp lies $\bar{\sigma} \subset U_{x} \cap S_{x}$, and

(c) $\bar{\sigma} \cap B \neq \varnothing, B \in \Sigma^{0}$, implies $\bar{\sigma} \subset W_{B}$.

For any free simplex $\sigma$ of $K(n)$ (i.e. not a face of any other simplex) let $\phi(\sigma) \in \Sigma^{0}$ be defined by:

(d) if $\bar{\sigma} \cap B \neq \varnothing$ for some $B \in \Sigma^{0}$ (unique by (c)), let $\phi(\sigma)=B$,

(e) if $\bar{\sigma} \cap B=\varnothing$ for all $B \in \Sigma^{0}$, and $\bar{\sigma} \cap \bar{V} \neq \varnothing$, let $\phi(\sigma)=L(\bar{V} \neq \varnothing$ implies $L \in \Sigma^{0}$ ),

(f) otherwise there is at least onc $x \in A$ with $\bar{\sigma} \cap \bar{V}_{x} \neq \varnothing$, and let $\phi(\sigma)=\{x\} \in \Sigma^{0}$ for one such $x \in A$.

Then for each $B \in \Sigma^{0}$, let $K_{B}$ be the closed subcomplex of $K(n)$ which is the union of all $\bar{\sigma}$ for all free simplices $\sigma$ of $K(n)$ with $\phi(\sigma)=B$.

By choice, (1) is satisfied. Since $K_{B}$ contains all simplices meeting $B, K_{B}$ is a neighborhood of $B$ in $K$, and $K_{B} \subset U$ if $B=L, K_{B} \subset U_{x}$ if $B=\{x\}, x \in A$, so (2) is satisfied. Since for all free simplices $\sigma$ of $K(n), \bar{\sigma}$ is contained in some $K_{B}$, (3) is satisfied. For $B \neq B^{\prime}$ in $\Sigma^{0}$, the sets $K_{B}$ and $K_{B^{\prime}}$ intersect only along boundary points, giving (4).

The set $K^{1}$ is then defined to be the set of points belonging to two or more of the sets $K_{B}$ for $B \in \Sigma^{0}$. Thus (5) is satisfied, and further $K^{1}$ is a closed subcomplex of $K(n)$, with $\operatorname{dim} K^{1}<\operatorname{dim} K$.

Having iterated the procedure completes the lemma except for the proof of (6). Suppose then that $B \in \Sigma^{i}, C \in \Sigma^{j}, j>i$, and $K_{B} \cap K_{C} \neq \varnothing$, and let $x \in K_{B} \cap K_{C}$. Since $x \in K^{j}, x$ belongs to all sets $K^{k}$ for $i \leqq k \leqq j$, and by (3), there exists a $C_{k} \in \Sigma^{k}, C_{i}=B, C_{j}=C$, with $x \in K_{C_{k}}$. It then suffices to prove (6) when $j=i+1$ since one would then have $f(C) \leqq f\left(C_{j-1}\right) \leqq \cdots \leqq f\left(C_{i+1}\right) \leqq f(B)$. Thus, suppose $j=i+1$. By choice, $C=\{y\}$ for $y \in K^{i+1}$ and $K_{C} \subset U_{y} \cap S_{y}$, so $x \in S_{y}$. Now $K^{i+1}$ is a subcomplex of $K^{i}(n)$ for some $n$, as is also $K_{B}$. Thus $K^{i+1} \cap K_{B}$ is a subcomplex containing $x$, so $K_{B}$ contains any simplex of $K^{i+1}$ which contains $x$. Since $x \in S_{y}, x$ belongs to a simplex of $K^{i+1}$ which contains $y$, and hence $y \in K_{B}$. Thus $f(C)=f(y) \leqq f(B)$ by $(2)$.

Lemma 3. Let $K, L, F, p$, and $f$ be as in Lemma 2. Then there exists a finite collection $\Sigma=\Sigma^{0} \cup \cdots \cup \Sigma^{r}$ of sets $B \subset K$ such that:

(1) $f$ is constant valued on each $B \in \Sigma$;

(2) $L \neq \varnothing$ implies $L \in \Sigma^{0}$; 
and there exist disjoint subsets $T_{B} \subset K$, for $B \in \Sigma$, such that:

(3) $K$ is the union of the sets $T_{B}, B \in \Sigma$;

(4) $B \subset T_{B}$;

(5) $\bar{T}_{B} \subset\{z \in K \mid f(z) \leqq f(B)\}$;

(6) if $B \in \Sigma, T_{B}$ is an open subset of $Z^{i}=K-\bigcup T_{C}$, the union being for $C \in \Sigma^{j}, j>i$; and

(7) $T_{B} \cap \bar{T}_{C} \neq \varnothing$ implies $f(C) \leqq f(B)$.

Proof. Let $\Sigma=\Sigma^{0} \cup \cdots \cup \Sigma^{r}$ be given by Lemma 2, giving (1) and (2) by choice of $\Sigma$.

The sets $K_{B}, B \in \Sigma^{r}$, are disjoint closed sets of $K$ (since $K^{r+1}=\varnothing$ ) and they have disjoint open neighborhoods $V_{B}$ in $K$. Let $W_{B}$ be an open set of $K$ containing $K_{B}$ such that:

(a) $W_{B} \subset\{z \in K \mid f(z) \leqq f(B)\}$ (by Lemma 2, (2)),

(b) $W_{B} \cap K_{C} \neq \varnothing, C \in \Sigma$, implies $K_{B} \cap K_{C} \neq \varnothing$ (by normality of $K$ ),

(c) $W_{B} \cap C \neq \varnothing, C \in \Sigma$, implies $C=B$ (for by Lemma 2, (2), (4) and (5), the sets $C \in \Sigma^{i}$ and $K_{B}, B \in \Sigma^{j}$ are disjoint if $i \leqq j$ ), and

(d) $W_{B} \subset V_{B}$.

Then let $T_{B}, B \in \Sigma^{r}$, be an open set containing $K_{B}$ with $\bar{T}_{B} \subset W_{B}$.

Inductively, suppose $T_{B}$ has been defined for $B \in \Sigma^{j}, j>m$, satisfying:

(a') $K^{j} \subset \bigcup T_{C}$, the union being for $C \in \Sigma^{k}, k \geqq j$,

(b') $B \subset T_{B}$,

(c') $\bar{T}_{B} \subset\{z \in K \mid f(z) \leqq f(B)\}$,

(d') If $B \in \Sigma^{j}, T_{B}$ is an open subset of $Z^{j}$ (see (6)) containing $Z^{j} \cap K_{B}$, and

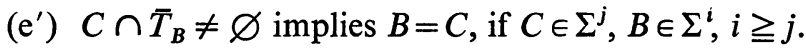

This is clear for $m=r-1$, with the choices made for $T_{B}, B \in \Sigma$ ?

Then define sets $T_{B}, B \in \Sigma^{m}$, as follows. $Z^{m}$ is a closed subset of $K$, having been formed by removing open sets from $Z^{m+1}$, and by $\left(\mathrm{e}^{\prime}\right)$, the sets $C \in \Sigma^{t}, t \leqq m$, are contained in $Z^{m}$, so the sets $K_{B} \cap Z^{m}, B \in \Sigma^{m}$, are nonempty. By $\left(\mathrm{a}^{\prime}\right)$ and Lemma 2:5), the sets $K_{B} \cap Z^{m}$ are disjoint closed subsets of $Z^{m}$, and let $V_{B}$ be disjoint open neighborhoods in $Z^{m}$ of the sets $K_{B} \cap Z^{m}$. Then let $W_{B}$ be an open set of $Z^{m}$ containing $K_{B} \cap Z^{m}$ and such that:

(1) $W_{B} \subset\{z \in K \mid f(z) \leqq f(B)\}$, (by Lemma 2,(2));

(2) $W_{B} \cap K_{C} \neq \varnothing$ implies $Z^{m} \cap K_{B} \cap K_{C} \neq \varnothing$ (normality);

(3) $W_{B} \cap C \neq \varnothing$ implies $C=B$ (for $W_{B} \cap C \neq \varnothing$ implies $C \in \Sigma, i \leqq m$, by (a'), and so $C$ and $K_{B}$ are disjoint if $i<m, C$ and $K_{B} \cap Z^{m}$ are disjoint for $i=m$ );

(4) $W_{B} \cap \bar{T}_{C} \neq \varnothing$ implies $\bar{T}_{C} \cap K_{B} \cap Z^{m} \neq \varnothing$ (normality);

(5) $W_{B} \subset V_{B}$.

Then let $T_{B}$ be open in $Z^{m}$, containing $K_{B} \cap Z^{m}$ with $\bar{T}_{B} \subset W_{B}$.

Properties $\left(\mathrm{a}^{\prime}\right)-\left(\mathrm{d}^{\prime}\right)$ are then trivial for $j>m-1$, and $\left(\mathrm{e}^{\prime}\right)$ is trivial except when $B \in \Sigma^{m}$. If $C \cap \bar{T}_{B} \neq \varnothing, C \in \Sigma$, then $C \in \Sigma^{i}$ for $i \leqq m$ by $\left(\mathrm{a}^{\prime}\right)$; and then by (3) in the choice of $W_{B}, C=B$. 
By induction, the sets $T_{B}$ are defined for all $B \in \Sigma$, property (3) of the lemma is just $\left(a^{\prime}\right),(4)$ is $\left(b^{\prime}\right),(5)$ is $\left(c^{\prime}\right),(6)$ is part of $\left(d^{\prime}\right)$, leaving only property (7) to be proved. Suppose then that $B, C \in \Sigma$ with $T_{B} \cap \bar{T}_{C} \neq \varnothing$, with $B \in \Sigma^{i}, C \in \Sigma^{j}$. If $j<i, T_{C} \subset Z^{j}$ and does not meet $T_{B}$ by (6). If $j=i$, the sets $T_{B}$ and $T_{C}$ are disjoint by $T_{B} \subset W_{B} \subset V_{B}$. Thus $j>i$, and by property (4) of $W_{B}, T_{C} \cap K_{B} \cap Z^{i} \neq \varnothing$, so $T_{C} \cap K_{B} \neq \varnothing$ and $W_{C} \cap K_{B} \neq \varnothing$. By property (2) of $W_{C}, K_{C} \cap K_{B} \cap Z^{j} \neq \varnothing$, so $K_{B} \cap K_{C} \neq \varnothing$ with $B \in \Sigma^{i}, C \in \Sigma^{j}$ and $j>i$. By (4) of Lemma 2, this gives $f(C) \leqq f(B)$, giving property (7).

THEOREM 7. Let $K$ be a finite simplicial complex, L a closed subcomplex, $F$ a finite space, $p \in F$, and $\mathfrak{A}$ the space of continuous functions $h:(K, L) \rightarrow(F, p)$ with the compact open topology.

(a) If $f \in \mathfrak{A}$, there exists a $g \in \mathfrak{U}$ such that $\{h \in \mathfrak{U} \mid h \leqq g\}$ is a neighborhood of $f$ in $\mathfrak{A}$.

(b) If $f, f^{\prime} \in \mathfrak{U}$ are homotopic (relative to $L$ ), there exist elements $\phi_{i} \in \mathfrak{U}$, $0 \leqq i \leqq s$, with $\phi_{0}=f, \phi_{s}=f^{\prime}$, and for $0 \leqq i<s$, either $\phi_{i} \leqq \phi_{i+1}$ or $\phi_{i} \geqq \phi_{i+1}$.

Proof. With the notation of Lemma 3, each $x \in K$ belongs to a unique $T_{B}$, $B \in \Sigma$, and let $g(x)=f(B)$.

If $L \neq \varnothing, L \in \Sigma$, and $L \subset T_{L}$ so $g(x)=f(L)=p$ for all $x$ in $L$

$g$ is continuous. If $x \in K, x \in T_{B}$ say, and let $C_{1}, \cdots C_{n} \in \Sigma$ be the sets $C$ for which $x \in \bar{T}_{C}, x \notin T_{C}$. There is then a neighborhood $N$ of $x$ with $N \cap T_{A} \neq \varnothing$ only if $A \in\left\{B, C_{1}, \cdots, C_{n}\right\}$. For $z \in N, g(z)$ takes one of the values $f(B)$ or $f\left(C_{i}\right)$. Since $T_{B} \cap \bar{T}_{C_{i}} \neq \varnothing, f\left(C_{i}\right) \leqq f(B)=g(x)$ by (7) of Lemma 3. Thus there is a neighborhood $N$ of $x$ with $z \in N$ implying $g(z) \leqq g(x)$, and hence $g$ is continuous.

Thus $g \in \mathfrak{A}$.

Let $\mathfrak{B}=\left\{h \in \mathfrak{X} \mid h\left(\bar{T}_{B}\right) \subset U_{f(B)}\right.$ for all $\left.B \in \Sigma\right\} . \mathfrak{B}$ is open in $\mathfrak{A}$ for each $\bar{T}_{B}$ is compact and each $U_{f(B)}$ is open. Then $f \in \mathfrak{B}$, for if $x \in \bar{T}_{B}, f(x) \leqq f(B)$ by (5) of Lemma 3 . If $h \in \mathfrak{B}, x \in K$, then $x \in T_{B}$ for some $B$ and since $h \in \mathfrak{B}, h(x) \leqq f(B)=g(x)$. Thus $h \leqq g$, so $f \in \mathfrak{B} \subset\{h \in \mathfrak{A} \mid h \leqq g\}$, completing part (a).

Now let $H: K \times I \rightarrow F$ be a homotopy of $f$ and $f^{\prime}$ (rel $L$ ). Let $J \subset I$ be the set of $t \in I$ for which there is a finite set $\phi_{i} \in \mathfrak{A}, 0 \leqq i \leqq m$, with $\phi_{0}=f, \phi_{m}=H(, t)$ and such that for $0 \leqq i<m$ either $\phi_{i} \leqq \phi_{i+1}$ or $\phi_{i+1} \leqq \phi_{i}$. Clearly, $0 \in J$ by taking $\phi_{0}=f, m=0$. If $t \in J$ with corresponding elements $\phi_{i} \in \mathfrak{A}$, let $\phi_{m+1}$ be a map for which $\left\{h \mid h \leqq \phi_{m+1}\right\}$ is a neighborhood of $H(, t)$. There is then an $\varepsilon>0$ such that $|t-s|<\varepsilon$ implies $H(, s) \in\left\{h \mid h \leqq \phi_{m+1}\right\}$, so $\phi_{m+2}=H(, s)$ if $|t-s|<\varepsilon$ shows that $J$ contains a neighborhood of $t$. Thus $J$ is open. Similarly, if $t \in \bar{J}$, there is a $g$ such that $\{h \in \mathfrak{U} \mid h \leqq g\}$ is a neighborhood of $H(, t)$ and hence there is an $\varepsilon>0$ such that $|t-s|<\varepsilon$ implies $H(, s) \leqq g$. Since $t \in J^{\prime}$ c there is a $t^{\prime} \in J$ with $\left|t-t^{\prime}\right|<\varepsilon$, and so elements $\phi_{j} \in \mathfrak{A}, 0 \leqq j \leqq n, \phi_{n}=H\left(, t^{\prime}\right)$ which give $t^{\prime} \in J$. Letting $\phi_{n+1}=g, \phi_{n+2}=H(, t)$, one has $t \in J$ so $J$ is closed. Thus $J=I$ and (b) is proved. 
Corollary 5. Homotopy classes of maps $f:(K, L) \rightarrow(F, p)$ are in 1-1 correspondence with the components of $\mathfrak{A}$, i.e. path components and components coincide in $\mathfrak{A}$.

\section{REFERENCES}

1. P. Alexandroff, Diskrete Räume, Mat. Sb. (N.S.) 2 (1937), 501-518.

2. S. Eilenberg and N. Steenrod, Foundations of algebraic topology, Princeton Univ. Press, Princeton, N. J., 1952.

3. R. H. Fox. On topologies for function spaces, Bull. Amer. Math. Soc. 51 (1945), 429-432.

4. S. T. Hu, Homotopy theory, Academic Press, New York, 1959.

5. J. L. Kelley, General topology, Van Nostrand, Princeton, N. J., 1955.

6. M. C. McCord, Singular homology groups and homotopy groups of finite topological spaces, (to appear).

MATHEMATICAL INSTITUTE, OXFORd UNIVERSITY, OXFORD, ENGLAND 